\title{
STUDIES OF AURORAL ARCS USING FREJA SATELLITE AND GROUND-BASED DATA
}

\author{
H. U. Frey,* G. Haerendel,* J. Clemmons,* D. D. Wallis,** \\ J. Vogt, ${ }^{*}$ O. H. Bauer, ${ }^{*}$ E. Rieger, ${ }^{*}$ M. H. Boehm* and \\ H. Lühr*** \\ * Max-Planck-Institut für extraterrestrische Physik, 85740 Garching, \\ Germany \\ ** National Research Council, Ottawa, Canada KIA OR6 \\ *** Technische Universität, 38106 Braunschweig, Germany
}

\begin{abstract}
During two Freja passes over auroral arc systems special wide-angle CCD cameras were operated from the ground. Comparisons of the optical observations with satellite-measured plasma parameters are performed. Motions of fine structures of the arcs are interpreted in terms of high-altitude electric fields shielded from the lower ionosphere. Independent measurements of current density, accelerating voltage and energy flux are evaluated and show good agreement.
\end{abstract}

\section{INTRODUCTION}

The instruments of the joint Swedish/German scientific satellite Freja have been designed to give high temporal and spatial resolution measurements of the auroral plasma between the auroral particle acceleration region and the lower ionosphere $/ 1 /$. The scientific objective is to study the interaction between the hot magnetospheric plasma and the topside atmosphere.

The Max-Planck-Institut für extraterrestrische Physik (MPE) developed a low-light-level CCD camera system aiming for high time-resolution and wide-angle images of the aurora. In February / March 1993 the two cameras had been set up near Gillam/Manitoba $\left(56.4^{\circ} \mathrm{N}, 265.4^{\circ} \mathrm{E}\right)$ at sites separated by $35 \mathrm{~km}$ for stereoscopic imaging. Out of several observations in the evening sector ( 21 MLT) we selected two nearly overhead passes of Freja over auroral arcs with a typical inverted-V electron spectrum and with small moving structure modulations.

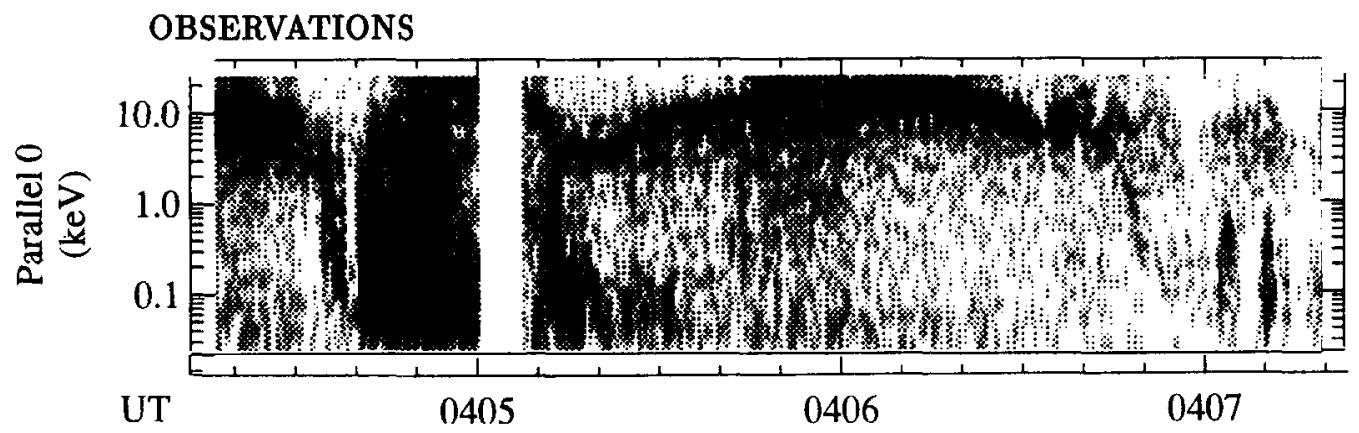

Fig. 1. Energy-time spectrogram of magnetic field-aligned electrons on 18 Mar 1993.

The Freja passage on $18 \mathrm{Mar} 1993$ (orbit 2152) occurred a few minutes after an auroral breakup starting at 03:57 UT. The aurora showed very dynamic changes until 04:04 UT. Later a broad band with brightness modulations of up to $30 \%$ developed. Due to the limited extent of our field of view in the north-south direction $(\sim 125 \mathrm{~km})$ we only saw the equatorward part of this band. The brightness patterns changed shape and moved coherently toward the west with speeds between 1.3 and $2.8 \mathrm{kms}^{-1}$. Our stereoscopic observations enabled the determination of the height of the lower edge of these patterns which was $104 \pm 5 \mathrm{~km}$. At about 04:05 UT Freja had passed through a flow reversal region as observed by the BARS radar maps and by the magnetograms of the CANOPUS system. The broad band exhibits the typical inverted-V signature between 04:05:20 and 04:06:40 UT with a fairly isotropic distribution and very few low-energy electrons (Fig. 1). 
Fig. 2. All-sky image taken at Rabbit Lake on 20 Mar 1993. (Courtesy of D. McEwen, University Saskatchewan). The Freja orbit between 03:12:00 and 03:21:00 is projected down to $100 \mathrm{~km}$ altitude. The position of Freja is shown by an asterisk.
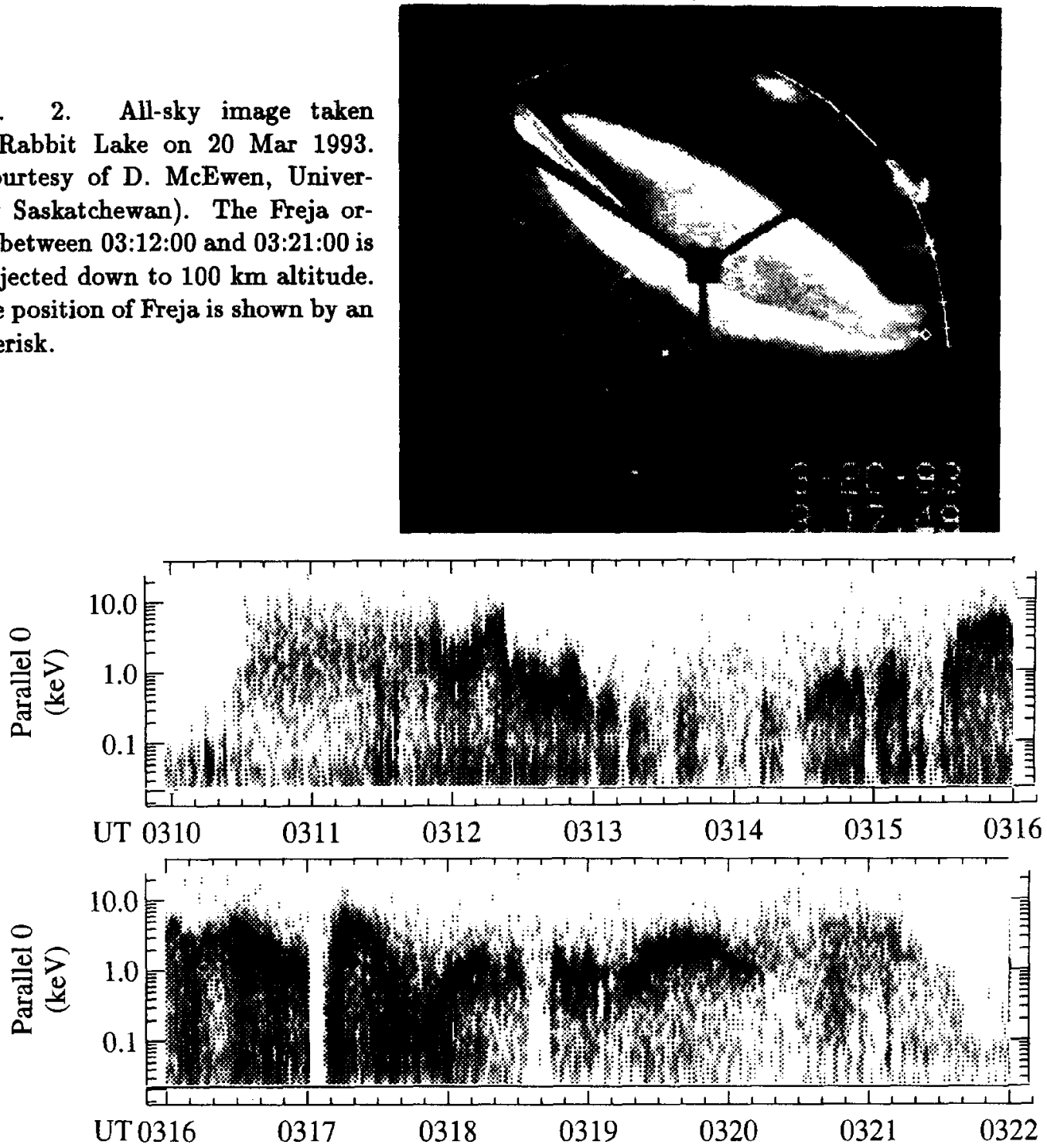

Fig. 3. Energy-time spectrogram of magnetic field-aligned electrons on $20 \mathrm{Mar} 1993$.

On 20 Mar 1993 (orbit 2178) the BARS radar and the CANOPUS magnetometer chain measured a flow reversal in the Ft. Churchill area $260 \mathrm{~km}$ north of Gillam. All-sky images from Rabbit Lake $(580 \mathrm{~km}$ west of Gillam, Fig. 2) show that Freja traversed a long east-west aligned arc system twice. South of the dominant arc we see diffuse weak aurora. North of that arc a dark gap is followed by a highly structured arc. Between 03:14 and 03:22 UT the dominant arc developed a central bright fold structure, the altitude of which was $125 \pm 8 \mathrm{~km}$. The folds moved eastward at speeds accelerating from 0.7 to $1.5 \mathrm{kms}^{-1}$. Clearly distinguishable on the real time images of our CCD cameras was a parallel fold system about $8 \mathrm{~km}$ south of the eastward drifting one, which moved in the opposite direction with similar speeds $\left(1.0-1.5 \mathrm{kms}^{-1}\right)$. The TESP electron spectrogram shows very different signatures (Fig. 3). First Freja passed a region with very diffuse electron spectra. When Freja passed the central arc system for the first time (03:11:50 03:13:00) the spectra were dominated by field aligned electrons of 2-6 keV. Then the satellite passed a region of very low electron fluxes of $0.05 \mathrm{ergcm}^{-2} \mathrm{~s}^{-1}$. The flow reversal region with highly structured field aligned electrons of high as well as low energy was passed by Freja between 03:15:30 and 03:18:30. Separated by an area without any significant electron flux and auroral emission (03:18:40) a region with mostly isotropic electrons of a typical inverted- $V$ signature and of energy fluxes of $1.6 \mathrm{ergcm}^{-2} \mathrm{~s}^{-1}$ followed. A careful examination of the electron spectrogram shows small modulations appropriate to the embedded folded arc. Later we again saw a region of diffuse electron fluxes corresponding to the diffuse aurora in the south. 


\section{DISCUSSION}

For the calculation of the field-aligned current density from Freja's magnetometer data we assumed that the local magnetic field is disturbed by current sheets aligned along the background field (IGRF model). The current density was then calculated from gradients in the perturbation field using Ampére's law. A minimum variance analysis /2/ was used to get the location and orientation of the sheets (Fig. 4). The current sheets are aligned along the auroral oval and the prominent arc apparently represents a broad east-west aligned sheet. Furthermore the gaps in the electron spectra at 03:15:22 and 03:18:32 UT could be identified as regions of downward field-aligned currents.

Fig. 4. Map of Canada with the projected Freja orbit between 03:08:00 and $03: 22: 00$ on 20 Mar 1993 and the current sheets calculated from the magnetometer data. The filled sheets belong to the main arc.

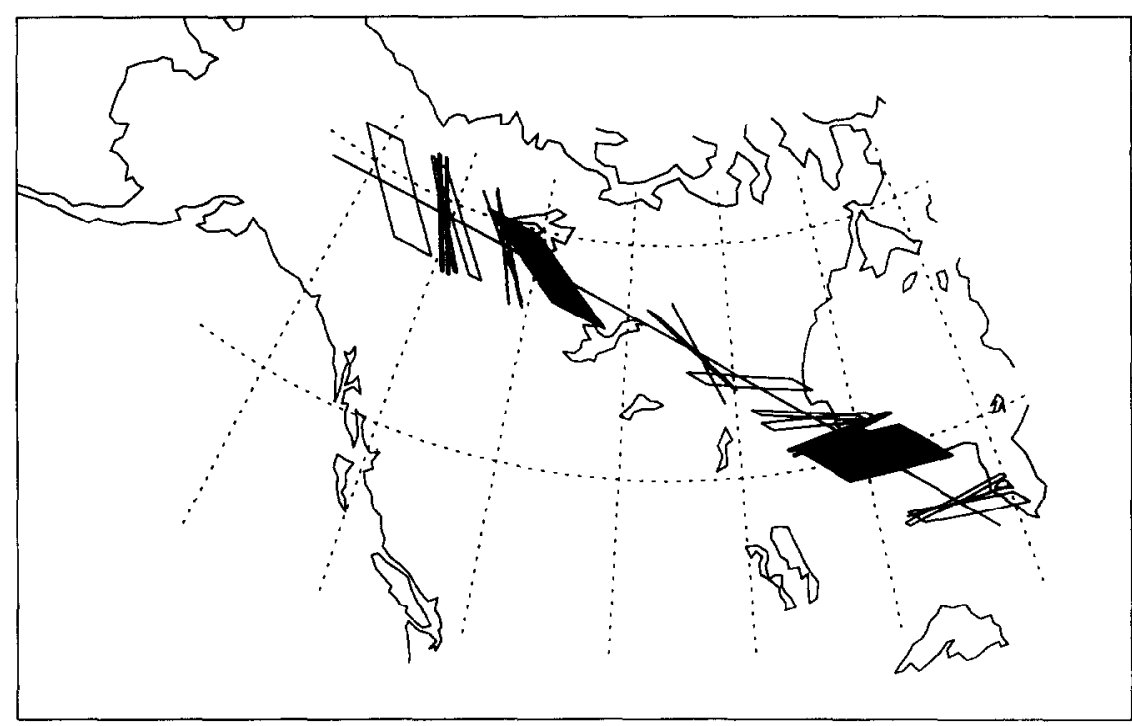

Theoretical calculations based on the height of the arcs yield primary electron energies of $12 \mathrm{keV}$ and $2.0 \mathrm{keV} / 3 /$. For March 20 this value is in very good agreement with the mean energy measured by Freja $(2.0 \mathrm{keV})$, but it is much lower for March 18 when Freja measured $25 \mathrm{keV}$. On March 20 there had been quiet geomagnetic conditions for more than 9 hours $\left(K_{p}=2+\right)$ and so the actual atmospheric composition should have been like the quiet model atmosphere. The observation on March 18 was made during much higher geomagnetic activity $\left(K_{p}=50\right.$ and $\left.K_{p}=5-\right)$ and shortly after the substorm. These conditions should result in a density enhancement of the higher atmosphere $/ 4 /$. Thus the electrons could not penetrate as deep as the quiet atmosphere model predicts.

Fig. 5. Downgoing electron energy flux measured with the TESP detector (left-hand scale) and auroral brightness contour along the projected satellite pass (right-hand scale) for 18 Mar 1993.

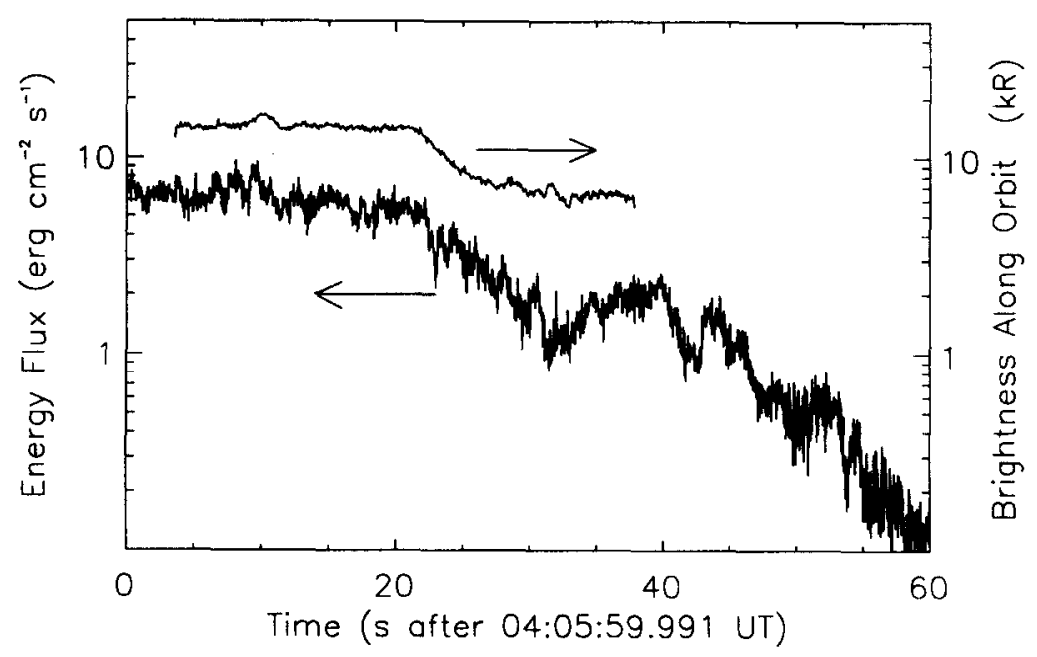

The westward drift of arc structures on March 18 would correspond to an ionospheric electric field of 65 to $140 \mathrm{mVm} \mathrm{m}^{-1}$, whereas the fold motion on March 20 would correspond to 35 to $75 \mathrm{mVm}^{-1}$. According to the BARS radar the electric field in this region was less than $20 \mathrm{mVm}^{-1}$. Therefore it appears that the fine-structures are not frozen in the ionospheric plasma and they may instead 
be caused by motions at higher altitudes where electric fields are shielded from the ionosphere by parallel potential drops. It is interesting to note that in both arcs the westward drift occurred on the equatorward edge of the arc. This behavior corresponds to the model of an inverted-V event with electric fields pointing into the arc on both sides.

The auroral CCD cameras of MPE have been calibrated at various wavelengths and thus allow us to deduce the primary electron energy fluxes from the brightness of the auroral arcs. Figure 5 contains the TESP-measured electron energy flux and the auroral emission at $557.7 \mathrm{~nm}$ at the magnetic foot-point of Freja at $100 \mathrm{~km}$ height taken out of the real-time images when Freja was within the field of view on March $18 / 5 /$. Both curves show very similar trends. The maximum brightnesses of the two inverted-V events were $15.2 \mathrm{kR}$ and $3.5 \mathrm{kR}$ at $557.7 \mathrm{~nm}$, respectively. Taking into account the mean primary energies of 25 and $2 \mathrm{keV}$ and the projection to the altitude of Freja, these measurements yield energy fluxes of 11.7 and $1.25 \mathrm{ergcm}^{-2} \mathrm{~s}^{-1}$ where the TESP electron sensor measured fluxes of 7.0 and $1.6 \mathrm{ergcm}^{-2} \mathrm{~s}^{-1}$, respectively.

Knowledge of the parallel potential drop, $\phi_{\|}$, of the parallel energy flux, $\dot{\varepsilon}_{\|}$, and of the current density, $j_{\|}$, offers the possibility of a comparison of the data. We assume that the energy flux is essentially a result of the conversion from magnetic to kinetic energy of the electrons.

$$
\dot{\varepsilon}_{\|}=j_{\|} \cdot \phi_{\|}
$$

\section{TABLE 1}

Calculation of inverted-V parameters. The first value of $\dot{\varepsilon}_{\|}$ stems from the particle, the second from the converted brightness measurements.

\begin{tabular}{|l|llll|}
\hline Date & Observed & \multicolumn{3}{l}{ Deduced } \\
& $\begin{array}{l}\phi_{\|} \\
{[\mathrm{kV}]}\end{array}$ & $j_{\|}$ & $\begin{array}{l}\dot{\varepsilon}_{\|} \\
{\left[\mu \mathrm{Am}^{-2}\right]}\end{array}$ & $\begin{array}{l}\phi_{\|}(\mathrm{Eq} \cdot 1) \\
{\left[\mathrm{ergcm}^{-2} \mathrm{~s}^{-1}\right]}\end{array}[\mathrm{kV}]$ \\
\hline $93-03-18$ & 25 & 0.42 & 7.0 & 16.7 \\
& & & 11.7 & 27.8 \\
$93-03-20$ & 2 & 0.54 & 1.6 & 2.9 \\
& & & 1.25 & 2.3 \\
\hline
\end{tabular}

The agreement between the accelerating voltage read from the observed energy spectra and the values calculated by means of equation (1) is quite good.

The arc on March 20 stretched over more than $2200 \mathrm{~km}$ covering a magnetic local time from 17:30 MLT to 21:20 MLT. The data measured by Freja in this inverted-V event during the two passes show that the local differences of the plasma parameters are not too big if we additionally take into account the time difference of $\sim 8$ minutes. The most remarkable optical feature is the difference between the smooth inverted- $V$ arc and the very much structured arc in the region of flow reversal. The mostly isotropic electrons in the inverted- $\mathrm{V}$ events indicate the electrostatic acceleration of a high temperature magnetospheric source population. The burst-like low energy electrons in the flow reversal regions can instead be interpreted as the signature of a wave acceleration.

\section{CONCLUSIONS}

Combined observations of inverted-V events by the Freja satellite and ground-based optical measurements show that the fine-structure in inverted- $V$ events seems to originate from modulations of the accelerating field-aligned potential at the interface between the topside ionosphere and hot magnetosphere. The comparison of ground-based and satellite-measured plasma parameters yielded similar results.

\section{REFERENCES}

1. R. Lundin and G. Haerendel, The Freja satellite mission, Eos Trans., 74, 321-324 (1993).

2. B.U.O. Sonnerup and L.J. Cahill Jr., Magnetopause structure and attitude from Explorer 12 observations, J. Geophys. Res., 2, 171-183 (1967).

3. M.H. Rees, Auroral ionization and excitation by incident energetic electrons, Planet. Space Sci., $\underline{11}, 1209-1218$ (1963).

4. CIRA 1972, Akademie-Verlag, Berlin, 1972.

5. G. Haerendel, H.U. Frey, O.H. Bauer, E. Rieger, J. Clemmons, M.H. Boehm, D.D. Wallis, H. Lühr, Inverted-V events simultaneously observed with the Freja satellite and from the ground, Geophys. Res. Lett., 21, 1891 (1994).

6. M.H. Rees, D. Luckey, Auroral electron energy derived from ratio of spectroscopic emissions 1. Model computations, J. Geophys. Res., 79, 5181 (1974). 